\title{
La réception des Provinciales dans les Mémoires du temps
}

\section{Emmanuèle Lesne-Jaffro}

\section{(2) OpenEdition}

\section{Journals}

Édition électronique

URL : http://journals.openedition.org/ccibp/570

DOI : $10.4000 /$ ccibp. 570

ISSN : 2493-7460

Éditeur

Centre international Blaise Pascal

\section{Édition imprimée}

Date de publication : 15 janvier 1996

Pagination : 3-8

ISSN : 0249-6674

\section{Référence électronique}

Emmanuèle Lesne-Jaffro, «La réception des Provinciales dans les Mémoires du temps », Courrier du

Centre international Blaise Pascal [En ligne], 18| 1996, mis en ligne le 07 janvier 2016, consulté le 20 avril 2019. URL : http://journals.openedition.org/ccibp/570 ; DOI : 10.4000/ccibp.570

Ce document a été généré automatiquement le 20 avril 2019

Centre international Blaise Pascal 


\title{
La réception des Provinciales dans les Mémoires du temps
}

\author{
Emmanuèle Lesne-Jaffro
}

1 Si l'on interroge les Mémorialistes du dix-septième siècle, c'est-à-dire des témoignages de contemporains, sur l'écho qu'ont pu produire les Lettres Provinciales, on ne peut manquer d'être d'abord frappé par le silence relatif dont cette affaire est entourée. La question de l'écho des Provinciales s'en trouve donc singulièrement relativisée, mais seulement dans l'exacte mesure où l'objet des Mémoires n'est pas de consigner tous les événements d'une époque: il s'agit soit de rapporter ce qui a pu avoir trait à la vie particulière du mémorialiste ou ce qu'il juge digne d'intéresser sa famille et éventuellement la postérité en complétant les lacunes de l'historiographie officielle. Qu'une querelle d'origine religieuse, même si elle prend précisément pour témoin le monde, soit passée sous silence dans des Mémoires n'a, à la réflexion, rien de très étonnant. Les mémorialistes ne relatent pas en général leurs lectures, ni les débats d'idées. L'année 1656 se passe pour beaucoup de mémorialistes en campagne militaire, pour d'autres l'événement marquant est la visite extraordinaire de Christine de Suède en France, ou quelque galanterie du jeune Louis XIV.

Pourtant une œuvre contemporaine de leur rédaction, Les Historiettes de Tallemant des Réaux, décrites par leur auteur comme de "petits Mémoires ", y consacre quelques lignes et promet d'en dire plus, mais l'ouvrage projeté ne verra malheureusement jamais le jour. Voici ce qu'il écrivit à propos de Pascal :

Sa sœur, religieuse à Port-Royal de Paris, luy donna de la familiarité avec les Jansénistes: il le devint luy-mesme, et c'est luy qui a fait ces belles lettres au Provincial que toute l'Europe admire, et que M. Nicole a mises en latin ${ }^{1}$.

La renommée des Provinciales semble bien être assurée, hors même des frontières de la France. L'éloge est avant tout esthétique, mais constitue peut-être une approbation de leur contenu.

Dans sa correspondance, $\mathrm{M}^{\text {me }}$ de Sévigné évoque à deux reprises Les Provinciales, en 1656 et en 1689. Le premier témoignage est donc saisi sur le vif et relate la réaction de l'épistolière à la lecture de la onzième lettre : «J'ai lu avec beaucoup de plaisir, écrit-elle à Ménage le 12 septembre 1656, la onzième lettre des jansénistes. Il me semble qu'elle est 
fort belle. Mandez-moi si ce n'est pas votre sentiment. Je vous remercie de tout mon cœur du soin que vous avez eu de me l'envoyer avec tant d'agréables choses. Cela divertit extrêmement en tous lieux, particulièrement à la campagne ${ }^{2}$. "

5 Le point de vue esthétique prévaut ici encore et la lettre est lue comme un divertissement plaisant. Notons que les Lettres sont bien diffusées en province. Qu'en est-il trente années plus tard, en 1689, lorsqu'elle évoque à nouveau les Petites Lettres? Voici en quels termes elle en relate la lecture :

Quelquefois pour nous divertir nous lisons les Petites Lettres. Bon Dieu, quel charme ! et comme mon fils les lit ! Je songe toujours à ma fille et combien cet excès de justesse de raisonnement serait digne d'elle, mais votre frère dit que vous trouveriez que c'est toujours la même chose. Ah, mon Dieu! tant mieux Peut-on avoir un style plus parfait, une raillerie plus fine, plus naturelle, plus délicate, plus digne fille de ces dialogues de Platon qui sont si beaux? mais après les dix premières lettres, quel sérieux, quelle solidité, quelle force, quelle éloquence, quel amour pour Dieu et pour la vérité! quelle manière de la soutenir et de la faire entendre ne trouve-t-on point dans les huit dernières lettres, qui sont sur un ton tout différent! Je suis assurée que vous ne les aviez jamais lues qu'en courant, grappillant les endroits plaisants, mais ce n'est point cela quand on les lit à loisir.

6 Madame de Sévigné a donc lu les Lettres au fil de leur publication, les unes après les autres, si elle les a lues toutes; trente ans après, elle les relit, sous la forme d'un recueil cette fois. Ce sont donc deux lectures différentes que son témoignage présente. Une troisième se dessine en creux: celle de sa fille, lectrice pressée de lire seulement les pointes. L'enthousiasme de $\mathrm{M}^{\mathrm{me}}$ de Sévigné, encore timide en 1656 , et franc en 1689 , se nourrit du contraste opposant les dix premières Lettres et leur ton de raillerie aux huit dernières, plus graves. La réussite rhétorique des Provinciales ici saluée, parce qu'elle joue sur plusieurs registres et l'éloge prévient ainsi une des critiques majeures des jésuites, celle de la disconvenance de la matière et du ton. De simple "plaisir» ou «divertissement» littéraire dont l'approbation révèle la prise de position dans la polémique, les Lettres sont devenues notamment par la force que leur confère la présentation en recueil, une œuvre rigoureuse au message spirituel irréfutable.

7 Qu'en est-il des témoignages des mémorialistes de l'époque? Les silences de l'abbé Arnauld, de Pontis, de Robert Arnauld, de Lancelot au sujet des Provinciales sont assez étonnants. On peut soupçonner chez l'abbé Arnauld quelques coupes du manuscrit, invérifiables en l'absence de manuscrit original, et une prudence dictée par la date de rédaction, qui correspond à la paix de l'Église. Mais on peut tout de même remarquer que le ton scandalisé du mémorialiste évoquant les persécutions des religieuses ne laisse guère de place à la réflexion sur l'épisode des Provinciales ou à un jugement sur leur effet.

8 Les mémoires d'une des héroïnes de la Fronde, alors en exil, font en revanche allusion aux Provinciales. Mademoiselle de Montpensier évoque assez longuement une visite qu'elle fit à l'abbaye de Port-Royal en 1657 ; l'affaire des Provinciales est encore très vivante, à peine terminée. La cousine du Roi est très élogieuse à l'égard de Port-Royal, et tient à préciser la nature de la curiosité qui l'y amène; elle résume à grands traits les débuts de l'affaire, en se montrant très favorable aux jansénistes, très admirative devant leur activité intellectuelle mais surtout devant la simplicité et la profondeur de leur piété :

Dans leur œuvres, écrit-elle, ils y portaient la pénitence plus loin pour les gens du monde que ne font d'ordinaire les religieux, qui en ont plus affaire que ces messieurs-là, et qui par là ménagent quelquefois plus leurs intérêts que les consciences de leur prochain. Cela déchaîna particulièrement les jésuites contre 
eux; qui les nommèrent jansénistes, comme on dirait les calvinistes, pour que ce nom, qui se rapporte à l'autre, effrayât d'eux et les fît passer pour hérétiques.

Comme ce sont des questions de théologie, et qu'il n'appartient pas aux femmes d'en parler, ni même à beaucoup d'hommes, c'est à ceux à qui Dieu a donné le pouvoir et le caractère d'en connaître, à les décider. Mais pour les mœurs ce sont des gens admirables ils prêchent et ils écrivent avec la plus belle éloquence, font des ouvrages merveilleux à la gloire de l'Église et des saints. (...) Si leur doctrine est mauvaise, il faut espérer qu'avec de si bonnes mœurs, ils obtiendront par leurs prières les lumières nécessaires pour le connaître et pour la changer. Cette doctrine a donc fait grand bruit dans la Sorbonne, où l'on a condamné des propositions de Jansénius à quoi ils ont souscrit, et se sont soumis à l'Église et au Saint-Père avec le dernier respect. Cette dispute a causé beaucoup de scandale aux Huguenots, parce les jésuites ont écrit des lettres contre les jansénistes sur leur sévérité, et eux contre les jésuites sur leur relâchement en cela il y a eu peu d'esprit de charité3.

9 Mademoiselle de Montpensier fait ici directement allusions aux Provinciales et l'on peut penser que cette éloquence qu'elle admirait tout à l'heure chez les jansénistes, est en partie celle des Petites Lettres. Sa visite à Port-Royal en est sans doute une conséquence, ce qui indique de ce point de vue que l'enjeu des Provinciales est bien atteint. Mademoiselle se fait ensuite l'écho de bruits expliquant les origines de la haine des jésuites contre les jansénistes ${ }^{4}$ et finit par conclure que les jésuites ont eu tort de répondre aux attaques de ceux-ci, sous-entendu aux Provinciales. Si les Provinciales ne suscitent pas de commentaire particulier de la cousine du Roi, la visite charmée de celle-ci à Port-Royal en découle directement. Les Lettres ont donc apparemment atteint leur objet, en disposant les mondains à se laisser convaincre de la grande piété des solitaires et des religieuses de Port-Royal. Est-ce un hasard si Mademoiselle emploie le terme de charité, qui est précisément un des arguments de la défense par les jansénistes de la raillerie, et de la lettre 11 en particulier? Aurait-elle eu vent des débats et une connaissance un peu précise des Lettres? Il ne semble pas qu'elle les ait lues elle-même, mais elle est informée de la polémique.

10 Faute d'avoir repéré des allusions aux Provinciales chez d'autres mémorialistes mondains, nous nous attacherons maintenant à considérer les Mémoires issus des milieux très proches de Port-Royal, en utilisant comme contrepoint les prolixes Mémoires du Père Rapin.

11 Nous avons donc interrogé les Mémoires de Du Fossé, le Journal de Saint-Gilles, l'Histoire générale de Port-Royal de Clémencet, Les Mémoires sur l'histoire ecclésiastique du $\mathrm{XVII}^{\mathrm{e}}$ siècle d'Hermant qui constituent l'essentiel des remarques ou anecdotes concernant la publication des Provinciales. Le recueil d'Utrecht décalque presque mot à mot le texte de Clémencet sur l'affaire des Provinciales, je ne m'y arrêterai donc pas ici.

12 Quelques réflexions se repèrent chez Pontchartrain, Taigner, Deslyons, Lamy, Beaubrun, collationnés par Jean Mesnard dans le premier tome des CEuvres complètes de Pascal.

13 Deux ordres de réflexions se dégagent essentiellement de cette enquête. Il m'a paru intéressant dans un premier temps de repérer dans quels termes se formulent les éloges des Provinciales. Dans un second temps de noter quel est leur succès auprès du public, et comment ce public est désigné ou défini.

14 Si l'on se pose la question de la place accordée à l'épisode des Provinciales dans ces œuvres, on s'aperçoit aussitôt que c'est chez les jésuites que les Lettres sont commentées de la manière la plus détaillée. La réfutation des Lettres est minutieuse chez Rapin et occupe plus de cent pages de ses Mémoires. 

dans leur domaine, comme l'égal des commentaires de César, c'est-à-dire de la référence incontestable en matière d'éloquence naturelle. Le rapprochement est pour le moins surprenant, mais l'éloge fait flèche de tout bois. C'est la virtuosité des Provinciales qui est somme toute mise en avant. Les Lettres sont dites «agréable(s) », écrites de " manière la plus ingénieuse ». Les Lettres sont rapidement résumées les unes après les autres. Certaines anecdotes relatives aux péripéties de la clandestinité sont relatées, ainsi que les 
réponses de Pascal aux principaux griefs des jésuites. En conclusion, Clémencet, sans prendre directement l'opinion à son compte, constate :

Ces dix-huit lettres de M. Pascal ont toujours été regardées comme un chef d'œuvre. C'est de tous les ouvrages qui ont jamais été fait contre les jésuites celui qui leur a causé le plus de chagrin, parce qu'il les a mieux fait connaître et les a démasqués entièrement ${ }^{8}$.

Clémencet quitte ici l'éloge hyperbolique pour inscrire les Provinciales en tête des ouvrages de polémique anti-jésuites :

Il fallait un Pascal, qui traitant la matière avec cette vivacité merveilleuse, et cet heureux enjouement qu'il avait reçu de la nature, rendît à jamais les casuistes et leurs partisans l'objet de la risée et du mépris, non seulement de tous les gens de bien, mais de tout le genre humain. Tel fut l'effet des Lettres Provinciales'.

Le style est à l'origine du succès des Lettres. Dans la définition de cet heureux enjouement reçu de la nature, se perçoit un écho de la définition du sublime. Clémencet cite les propres paroles de Pascal rapportées par sa nièce, lorsqu'il justifie le choix d'un style railleur :

On m'a demandé, aurait dit Pascal, pourquoi j'ai employé un style agréable, railleur et divertissant ; je réponds que si j'avais écris d'un style dogmatique, il n'y aurait eu que les savants qui les auraient lues; et ceux-là n'en avaient pas besoin, en sachant pour le moins autant que moi là dessus. Ainsi j'ai cru qu'il fallait écrire d'une manière propre à faire lire mes lettres par les femmes et les gens du monde afin qu'ils connussent le danger de toutes ces maximes et de toutes ces propositions qui se répandaient alors ${ }^{10} \ldots$

Mademoiselle de Montpensier avait bien compris que les femmes ne pouvaient entrer dans les méandres des querelles de théologiens, et l'on a l'impression que Pascal lui répond lorsqu'il articule le choix du style choisi à la considération du destinataire. Il fallait plaire au public, le séduire.

Le journal de Saint-Gilles présente l'avantage d'une rhétorique plus personnelle que les écrits rédigés après coup et qui se nourrissent aux mêmes sources. D'autre part on y perçoit admirablement bien l'exacerbation progressive du conflit. Dans quels termes désigne-t-il les Lettres? L'adresse de la première lettre est soulignée, et le thème de leur agrément est repris. Les Lettres sont évoquées au fur et à mesure de leur parution, au fil des événements survenant à la Sorbonne et des interdictions qui frappent les imprimeurs. La seconde montre «clairement et galamment combien l'opinion, ou plutôt les diverses opinions des molinistes sont ridicules ». Cette lettre est qualifiée d'« excellente pièce », et « fait voir avec un agrément merveilleux l'injustice de la censure » qui frappe Arnauld ${ }^{11}$. À propos de la troisième lettre Saint-Gilles commente à son tour la brièveté de la forme et la loue :

On éprouve que ces petites pièces font beaucoup plus d'effet que les autres plus longues et plus considérables, car en peu de temps on y est agréablement instruit de la véritén

Le ton de Saint-Gilles se durcit au fil des jours. Les commentaires qu'il consigne ensuite dans son journal concernent avant tout les conditions de l'impression, et sa propre participation à l'impression des 7, 8, 9 et $10^{\mathrm{e}}$ Lettres $^{13}$.

29 À propos du style de Pascal, le Manuscrit Lamy, apporte de curieuses précisions, une étonnante caractérisation. Les Provinciales ne sont pas explicitement mentionnées, mais il est dans le contexte question de l'engagement de Pascal contre les jésuites : 
Sa facilité était merveilleuse à s'énoncer de vive voix et par écrit. Il avait pour but dans ce beau talent d'imiter tout à fait la nature. Pour y parvenir, il forma son style sur deux sortes de personnes auxquelles on ne s'était jamais avisé d'attribuer la justesse du langage. Le premier genre est des gentilshommes de campagne, dont le revenu peut aller de mille écus et dont l'éducation est naturelle, sans avoir passé par le tumulte d'un collège. Le second genre est des filles qui n'ont atteint que leur quinzième année. L'on peut juger si $\mathrm{M}$. Pascal a bien rencontré par le succès ${ }^{14}$. remarque, ce que rien n'interdit. C'est en somme une variante de la caractérisation par la "naïveté ». Un thème présent chez l'abbé Arnauld, et qui était un lieu commun que pratiquait la famille Arnauld, se rencontre ici, au sujet de la pureté de la langue lorsqu'elle est la langue de la famille et non une langue déformée et corrompue par le collège. témoignage est cette fois de la fin du dix-septième siècle. Il se fait l'écho des avis du public :

On n'avait encore rien vu de si fin, de si délicat et de si juste dans notre langue. Ceux qui connaissent les véritables règles de l'éloquence les voyaient parfaitement observées ; mais l'art y était tellement caché que la nature seule semblait avoir eu quelque part à ces riches productions. La raillerie en était si ingénieuse qu'elle faisait concevoir une juste indignation contre ceux qu'elle rendait ridicules, et les tours des pensées et des périodes y étaient si heureux qu'on relisait plusieurs fois avec plaisir comme des beautés toutes nouvelles les endroits qu'on avait lus d'abord avec admiration. Enfin ceux qui voulaient raisonner, parler et écrire noblement se proposaient ces Lettres comme des modèles, et les plus habiles étaient tout à fait persuadés que l'on ne pouvait pas se proposer en ce genre une plus parfaite idée du bon sens et de l'éloquence ${ }^{15}$.

Le caractère naturel du style, topos de la poétique classique, se trouve reformulé ici. Les Provinciales sont ensuite présentées comme un modèle d'argumentation digne d'être imité, ce qui rejoint l'expression rencontrée plus haut de « manuel des beaux esprits ».

Il est frappant de constater que le succès des Provinciales est d'abord présenté comme un succès de style, c'est-à-dire un succès littéraire et que ce succès conditionne le succès contre les adversaires. Admirer les Provinciales pour leur style, c'est être gagné à leur cause. Rapin ne s'y trompe pas lui-même, s'il se trompe sur l'identité de l'auteur des trois premières lettres attribuées à Arnauld, ce qui l'amène à tomber dans le piège du commentaire de style. En effet, il critique les trois premières lettres pour leur style en croyant viser Arnauld: «Il est vrai qu'Arnauld, tout grand génie qu'il était pour les sciences, n'avait rien de cet air délié que demande la line raillerie ${ }^{16} "$, et explique ainsi le passage du relai à Pascal plus apte à écrire, selon les propres termes de Rapin, «quelque chose de plus délicat et de plus piquant pour intéresser le public ». Mais si Rapin concède quelques talents à Pascal pour le style, il réserve ses attaques pour la jeunesse de ce dernier qu'il qualifie de libertine et pour son ignorance en matière de théologie. Il est bien obligé même de reconnaître que la cinquième Lettre « était pleine de certains traits d'une raillerie si délicate et d'une vivacité si fine que non seulement elle fut reçue du public avec bien plus d'applaudissements que les précédentes, mais qu'elle excita même une curiosité et une impatience pour les suivantes, dont on a peu d'exemples ${ }^{17}$ ». Admettre de telles qualités lorsqu'on est du camp des Jésuites est tout de même un tour de force. C'est reconnaître implicitement qu'en matière de style il est impossible de critiquer les Lettres.

Courrier du Centre international Blaise Pascal, 18 | 1996 
Cette bataille des Provinciales est bien une bataille pour conquérir le public; il faut donc plaire.

Voyons à présent quel succès les Lettres rencontrent et quel public elles touchent. Tallemant des Réaux a parlé d'un succès européen.

Rapin attaque immédiatement les Lettres en minimisant leur succès, prétendant qu'elles « n'eurent pas le succès qu'(Arnauld) s'en était promis ${ }^{18}$ ».

Il tente d'accréditer l'idée que la diffusion des Lettres était soigneusement orchestrée et le public préparé par la comtesse Du Plessis.

Comme elle prenait souvent l'empire, par la qualité de son esprit, sur ceux qui l'approchaient, ce fut à elle que l'on s'adressa à Port-Royal, où elle avait de grandes liaisons, afin qu'elle fit valoir les petites lettres auprès des beaux esprits, en les obligeant à en appuyer le succès de leur charges dans le monde, où ils s'étaient acquis tant de crédit. (...) Devant que la sixième lettre parut dans le public, on en envoya une copie à la comtesse pour la faire voir à ses amis (...) Elle leur dit qu'ils avaient trop d'esprit pour ne pas sentir eux-mêmes les beautés de ces lettres, pour lesquelles elle leur demandait leur protection (...). Après ce préambule la lettre fut lue, et elle ne pouvait pas manquer d'être admirée par des gens aussi disposés à plaire à la comtesse (...). Ils vont comme autant de trompettes publier par tout Paris que la sixième lettre au provincial commence à paraître, qu'elle était bien plus belle que celles qui avaient paru $^{19} \ldots$

Rapin est bien obligé de s'incliner devant le succès public des Lettres, mais il essaye de le faire passer pour de la manipulation. L'argument, répété souvent, se double ensuite d'un deuxième et enfin d'un troisième argument: le public est favorable à la critique des Lettres parce qu'il ne les examine pas véritablement, et en troisième lieu les Lettres trouvent grâce aux yeux d'un public de libertins qui trouvent chez les casuistes de quoi justifier leur libertinage. Deux publics se trouvent ainsi désignés, auxquels vient s'ajouter un troisième :

Après tout, cette fantaisie de sévérité, dont on avait fait à Port-Royal un des principes de la nouvelle opinion et qui était alors en vogue, ne laissait pas d'attirer des applaudissement à ces impostures parmi quelques honnêtes gens et parmi ceux qui faisant profession de probité, ont toujours de l'opposition au relâchement. C'est ce qui disposa les esprits simples à être favorables à ces faussetés sans les examiner et à en souhaiter la continuation, qui fut pareillement bien reçue de tous ceux qui vivaient dans quelques dérèglements, et surtout des femmes, qui trouvaient dans ces lettres de quoi autoriser leurs galanteries, par les opinions trop libres que Pascal attribuait faussement aux Jésuites, sans parler des rieurs et des fainéants de cour, qui ne cherchent qu'à se divertir et qu'il attira de son côtée ${ }^{20}$.

Après cette description édifiante du public des Provinciales qui manifeste bien à quel point Rapin ne peut contester le succès de l'œuvre, il finit par trouver un dernier argument : les Lettres n'ayant pas eu le succès escompté, il fallait un miracle et l'on utilisa celui de la Sainte Épine ${ }^{21}$. Trop d'arguments nuisent à l'argumentation.

Mais laissons Rapin et revenons aux milieux proches de Port-Royal qui ne comptent selon le jésuite que des "simples» des "ignorants", des "gens prévenus d'estime et d'inclination pour la belle morale » et enfin "tous ceux qui voulant bien être trompés n'approfondissent rien ».

Du Fossé avance l'idée (prêtée par Marguerite Perier à son oncle) que Les Provinciales étaient destinées à un public non initié au débat théologique, celui des femmes. Et la remarque citée plus haut de $\mathrm{M}^{\text {lle }}$ de Montpensier montre que ce public-là s'est reconnu. Selon Du Fossé, Pascal se proposait, afin d'empêcher la condamnation d'Arnauld, de : 
...faire voir le ridicule d'un tel procédé, et de mettre dans tout son jour la matière dont il s'agirait alors, afin que tout ce mystère étant découvert, et rendu, pour ainsi dire, sensible aux femmes mêmes, tout le monde se rassura dans la crainte qu'il pouvait avoir, qu'il ne s'agît dans cette affaire de quelque point de religion. C'est ce qu'il fit avec tant de netteté et d'agrément dans les Premières de ses Lettres, que tout Paris et que toute la France fut dans l'admiration de voir qu'on pût mettre dans une si grande évidence des questions si subtiles, et faire trouver tant d'agréments dans la lecture d'un ouvrage où elles sont discutées.

L'écho des Provinciales n'est évidemment pas seulement parisien, ce que confirme par ailleurs Saint-Gilles lorsqu'il précise à propos de la troisième qu'elle a "commencé (...) à paraître avec un éclat et un applaudissement encore plus grand que les deux précédentes. » et continue par cette remarque chiffrée: «On en a donné par Paris et envoyé dans les provinces par douzaines et le succès qu'on en apprend partout est incroyable». Saint-Gilles donne d'intéressantes indications chiffrées au sujet de la seconde. Il rapporte, à propos de la femme de l'imprimeur frappé d'interdiction une anecdote intéressante, relatant "qu'elle mit les formes quoique fort pesantes dans son tablier, passa en bas parmi les commissaire et gardes, et les porta chez un ami là auprès, où dès la nuit, on tira 300 de la seconde, et, le lendemain, 1200, ce qui irrite toujours de plus en plus les ennemis de la vérité, et surtout $M$. le Chancelier qui jette feu et flamme contre Monsieur Arnauld et ses amis, qu'il croit auteur de ces lettres. ${ }^{22}$ ”

Le secret de l'auteur était bien gardé, on le voit, puisqu'il a abusé jusqu'à Rapin, et il est considéré par Hermant comme une des raisons du succès des Lettres: "L'auteur de ces Lettres Provinciales s'était étudié sur toutes choses à demeurer inconnu (...). Mais il n'était presque pas possible qu'un ouvrage d'un si grand mérite n'excitât pas la curiosité des gens de lettres pour tâcher de découvrir de quelle main leur venait un présent si précieux $^{23}$.» Les Provinciales ont eu un très grand retentissement grâce au piquant de leur clandestinité :

Ces Lettres de M. Pascal eurent donc dans le public tout le bon succès qu'ils s'étaient proposés. Car outre qu'elle donnèrent à toutes les personnes non entêtées, la juste idée qu'elles devaient avoir des assemblées irrégulières de la faculté et du fruit qu'elles pouvaient produire, elles firent concevoir à tous les fidèles une sainte horreur de tant de maximes détestables de plusieurs casuistes modernes, qui voulaient passer dans l'Église pour des grands maîtres de morale ${ }^{24}$.

L'objet des Provinciales semble donc atteint, même si les premières n'ont pas réussi à éviter la condamnation d'Arnauld.

Pour Clémencet le succès des Provinciales va sans commentaires tant il est indiscutable : «Il est inutile de parler ici de l'accueil que le public fit à ces célèbres lettres, et aux suivantes (...). L'applaudissement fut général. Et l'estime qu'elles s'acquirent dès-lors, s'est non seulement soutenue, mais a encore augmenté. » Clémencet tient à souligner, en reprenant les termes de la onzième lettre que les Provinciales constituent un jeu pour la société avant un véritable combat, et il poursuit: «En effet ces lettres furent comme le signal d'un grand combat », ouvrant ainsi le champ aux Écrits des curés de Paris. Du Fossé distingue plusieurs publics successifs, au-delà de la Faculté, l'ensemble des évêques, et au-delà " toutes les personnes non entêtées ", soulignant toujours l'impatience du public. Il remarque en effet que le milieu des parlementaires est très attentif aux Lettres citant cette anecdote :

La première n'eut pas plutôt été lue qu'on aspirait avec impatience à voir la seconde et les suivantes. Rien n'était égal à l'empressement des premiers magistrats, par 
exemple M. de Bellièvre, alors premier président, qui donna ordre qu'on les lui apportât dans l'instant qu'elles paraîtraient ${ }^{25}$.

Les contours du public des Provinciales apparaissent ainsi avec netteté : les milieux théologiens et, parce que Pascal a su placer la bataille sur un plan littéraire, les milieux mondains. Il s'agit de diviser les premiers, d'y gagner des évêques, des curés. Dans les seconds, les parlementaires récents acteurs des troubles de la Fronde, paraissent aux premières loges, ainsi que les femmes puisque nous voyons intervenir à divers titres $M^{\text {lle }}$ de Montpensier, $\mathrm{M}^{\text {me }}$ de Sévigné, $\mathrm{M}^{\mathrm{me}}$ du Plessis. Ce n'est là sans doute qu'une des leçons très marginale de l'affaire des Provinciales, mais il semble bien qu'accessoirement elle manifeste tout de même, et là est peut-être un aspect du scandale, une tentative pour arracher les questions de religion aux seuls théologiens, en tous cas pour les rendre intelligibles à ceux qui constituent déjà l'opinion publique et en particulier aux femmes.

\section{NOTES}

1. Tallemant des Réaux, Les Historiettes, éd. A. Adam, Gallimard, La Pléiade, 1962, t. 2, p. 58.

2. Sévigné, Correspondance, éd. R. Duchêne, La Pléiade, Gallimard, 1972, t. 1, p. 40.

3. $\mathrm{M}^{\mathrm{lle}}$ de Montpensier, Mémoires, éd. Chéruel, t. III, p. 69.

4. Elle évoque la rancune des jésuites à l'égard d'un avocat, M. Marion, grand père de MM. Arnauld et Le Maître qui aurait plaidé contre eux lors d'une affaire liée à l'assassinat d'Henri IV.

5. Du Fossé, Mémoires pour servir à l'histoire de Port-Royal par M. Du Fossé, Utrecht, 1739, Livre I, chapitre XVII.

6. Ibid.

7. Clémencet, Histoire générale de Port-Royal depuis la réforme de l'abbaye jusqu'à son entière destruction, Amsterdam, J. Vanduren, 1756, t. 3, p. 443.

8. Ibid., p. 455-56.

9. Ibid., p. 456.

10. Ibid., p. 457.

11. Saint-Gilles, Journal, éd. E. Jovy, Paris, 1936, p. 117.

12. Ibid., p. 127-28.

13. Ibid., p. 180-81.

14. Manuscrit Lamy, cité par J. Mesnard dans les đEuvres Complètes de Pascal, Desclée de Brouwer, t. 1, p. 737.

15. Hermant, Mémoires, cités par J. Mesnard dans les Euvres Complètes de Pascal, Desclée de Brouwer, t. 1, p. 911.

16. Rapin R., Mémoires sur l'Église et la société, la cour, la ville et le jansénisme, 1644-69, éd. L. Aubineau, 1865, t. 2, p. 359.

17. Ibid., p. 366.

18. Ibid., p. 359.

19. Ibid., p. 367-68 et voir aussi p. 376.

20. Ibid., p. 398.

21. Ibid., p. 418.

22. Saint-Gilles, op. cit., p. 120.

23. Hermant, op. cit., t. 1, p. 911. 
24. Ibid.

25. Du Fossé, op. cit.

INDEX

Mots-clés : Pascal, Provinciales, réception, Montpensier (Mademoiselle de), Sévigné (Madame de), Port-Royal, mémoires, Arnauld (Antoine), Clémencet (Charles), Hermant (Godefroy)

\section{AUTEUR}

\section{EMMANUÈLE LESNE-JAFFRO}

CERHAC, Université Blaise Pascal 